Artículo ORIGINAL

\section{Fuerza de adhesión de brackets reacondicionados con diferentes técnicas adheridos repetidas veces en la misma superficie del esmalte}

Adhesion strength of brackets repeated bonding in the same surface of enamel and reconditioned with different techniques

\begin{abstract}
Resumen
Las fallas de adhesión y el reposicionamiento de brackets acontecen muy a menudo durante el tratamiento ortodóntico. Ante esta situación, el clínico puede optar por volver a adherir el mismo bracket del paciente o adherir uno nuevo. El estudio tuvo como objetivo determinar la variación de la fuerza de adhesión in vitro de brackets reacondicionados con las técnicas de flameado, microarenado y de brackets nuevos. 60 premolares sanas extraídos por indicación ortodóntica fueron agrupados aleatoriamente en 3 grupos: grupo I brackets reacondicionados con técnica de microarenado; grupo II: brackets reacondicionados con técnica de flameado y grupo III: brackets nuevos como control. Todos los brackets fueron adheridos con resina de autopolimerizado No Mix (Prime Dental Manufactuing INC). Inicialmente, brackets nuevos fueron adheridos a todos los grupos para determinar la fuerza de adhesión inicial (primer ensayo de cizallamiento); luego fueron sometidos hasta tres ensayos más utilizando una máquina universal de fuerzas. Los resultados mostraron que la fuerza de adhesión disminuyó en todos los grupos conforme al número de ensayos de cizallamientos, sin embargo, los brackets microarenados mostraron significativamente mayor fuerza de adhesión que los brackets flameados y nuevos. Aparentemente en el cuarto ensayo de cizallamiento se produjo un estrés máximo de la estructura del esmalte y, en consecuencia, la retención de la base de los brackets dejó de ser relevante.
\end{abstract}

\section{Abstract}

Bonding failures of brackets and the repositioning of them occur quite often during the orthodontic treatment. Upon this situation, the professional may choose to stick the same bracket or bond a new one. The purpose of this study was to evaluate changes of the bonding strength of reconditioned brackets with direct flame, microetching and new brackets attached several times on the same tooth surface in vitro. 60 healthy human premolars extracted for orthodontic reasons were randomly assigned to 3 groups: Group I reconditioned brackets with air abrasion; Group II: reconditioned brackets with flame; Group III: new brackets. All brackets were bonded with resin No Mix (Prime Dental Manufactuing INC). Initially, new brackets were bonded to all groups to determine initial bonding strength (first shear test), then were subjected to four tests using a universal testing machine. The results showed that bonding strength decreased in relation to the number of shear tests, however, reconditioned brackets with microetching showed significantly more adherence force than the new brackets and flames. Apparently, in the fourth share test maximum stress of occurred enamel so the retention of brackets base became irrelevant.

\section{Hugo Javier Luque Luque ${ }^{1}$ Luis Fernando Pérez Vargas ${ }^{2}$ Gregorio Javier Carhuamaca León ${ }^{3}$ Marco Antonio Coronado Tamaríz ${ }^{4}$

\author{
1,3,4 CD Práctica Privada \\ Dpto. Académico Estomatología Biosocial. \\ Facultad Odontología. UNMSM. Lima, Perú
}

Correspondencia:

CD. Hugo Javier Luque Luque

Dirección: Av. Michael Faraday 212, Urb. Sicuani Ate Teléfono: 326-3642 / 997673798

E-mail: hj1150@hotmail.com

Palabras clave: Adhesión. Brackets reacondicionados. Resistencia al cizallamiento.

Keywords: Bonding. Reaconditioned brackets. Shear bond strength.

\section{INTRODUCCIÓN}

Desde que Buonocore ${ }^{3}$ introdujo la técnica de grabado ácido en 1955, el concepto de adhesión comenzó a ser aplicado en todo el campo de la odontología. A diferencia de la odontología restaurativa que busca fuerzas de adhesión lo más perenne posible, la adhesión en ortodoncia es concebida en un periodo de tiempo, ya que los aditamentos como brackets permanecen adheridos a los dientes mientras dure el tratamiento ortodóntico. Aunque los avances de adhesión han permitido adherir satis- factoriamente dichos aditamentos en la superficie adamantina, las fallas de adhesión y el desprendimiento de brackets acontece muy a menudo. Esto puede deberse a fuerzas biomecánicas y/o masticatorias, poca retención de la base del bracket, del sistema adhesivo utilizado o falla en la técnica de adhesión.

Frente a un bracket despegado, el ortodoncista puede optar por volver a adherir el mismo bracket del paciente o adherir uno nuevo. El repegado del mismo es una opción muy escogida por los clínicos, no sólo porque disminuye los costos, sino también porque ahorra tiempo al no requerir una cita adicional para el paciente, esto acontece principalmente en los medios hospitalarios. Volver adherir un bracket también ocurre en casos de reposicionamiento de brackets $^{6}$.

Los métodos más utilizados para reacondicionar el bracket despegado son los del tipo inmediato realizado en el mismo consultorio; los métodos mediatos son realizados por empresas especializadas en reciclado, no muy frecuente en nuestro medio. El reacondiciona- 
miento con técnica de flameado es tal vez el método más usado para remover el adhesivo impregnado de la base del bracket. Por otro lado, la técnica de microarenado ha mostrado ser un buen método para reacondicionar el bracket, sin embargo, no es muy utilizado ni difundido por los clínicos.

Situaciones indeseables en donde el bracket se despega una, dos o hasta tres veces hacen mucho más crítico volver adherir un bracket. Si consideramos que en un tratamiento ortodóntico eficaz sólo están permitidos muy pocos errores, entonces, volver adherir un brackets debe garantizar una adecuada fuerza de adhesión a fin de evitar nuevas fallas y desconfort en el paciente. ¿Se debe entonces adherir un bracket nuevo o el mismo bracket despegado?

Es importante, por tanto, simular una situación clínica en donde brackets nuevos y brackets despegados reacondicionados son readheridos una, dos hasta tres veces para así conocer sus diferencias y variaciones en relación a su fuerza de adhesión.

\section{MATERIAL Y MÉTODOS}

\section{Recolección de los dientes}

60 dientes premolares con ápices cerrados, sin ningún tipo de lesión ni restauración, extraídos por indicación ortodóntica fueron recolectados y transportados en suero fisiológico evitando en todo momento su deshidratación. Luego de remover el tejido periodontal con curetas periodontales, fueron almacenados con agua destilada y saliva artificial "Salival" (proporción 1:2) en una heladera a una temperatura de $4{ }^{\circ} \mathrm{C}$ hasta el día de la prueba de cizallamiento ${ }^{8}$.

\section{Prueba mecánica de cizallamiento}

La superficie vestibular del diente se limpió con escobilla y pasta profiláctica a velocidad lenta durante 15 segundos; luego fueron enjuagadas durante 10 segundos. Las escobillas fueron sustituidas por nuevos por cada cinco dientes para mantener la misma acción mecánica de limpieza de todos los especímenes.

Los brackets fueron adheridos en la cara vestibular del diente a una distancia de $4 \mathrm{~mm}$ del plano oclusal con el sistema adhesivo de autocurado No Mix Prime Dental Manufacturing INC. Se siguió las indicaciones del fabricante.

Cada diente fue sumergido en bloques de acrílicos rotulados de $2 \mathrm{~cm}$ de diámetro interno por $2 \mathrm{~cm}$ de altura dejando solamente la corona del diente expuesta y la base del brackets paralela a los bordes del cilindro.

El ensayo se realizó en el laboratorio N. ${ }^{\circ} 4$ de la Facultad de Ingeniería Mecánica de la Universidad Nacional de Ingeniería (UNI-PERÚ) siguiendo las normas estandarizadas de la ISO TR $11405^{8}$. Se utilizó una máquina universal de fuerzas marca Amler año 2001 de procedencia americana. La prueba fue supervisada por el Ingeniero Mecánico Sebastián Lazo, Jefe del laboratorio N. ${ }^{\circ}$ 4 de dicha facultad.

Los bloques de acrílico con el diente embebido fueron colocados en una máquina universal de fuerzas con la cara vestibular y la base del bracket paralelo a la cizalla.

La máquina se programó a una velocidad de $1 \mathrm{~mm} / \mathrm{min}$. de acuerdo a la estandarización ISO que contempla velocidades de $0.75 \pm 0.30 \mathrm{~mm} / \mathrm{min}$, un rango de fuerza de carga de $0 / 500 \mathrm{~N}$ con una precisión de $\pm 0.1 \mathrm{~N}$. La herramienta de corte utilizada fue una cuchilla biselada la cual tomó contacto entre el diente y la base del bracket ${ }^{8}$.

La resistencia al cizallamiento fue registrado en $\mathrm{kg}-\mathrm{F}$ y luego convertido en $\mathrm{MPa}$ utilizando el área de la base del bracket $\left(12.33 \mathrm{~mm}^{2}\right)$. El área de la base del bracket fue determinada con un vernier digital de puntas secas marca Mitutoyo modelo 500-196.

\section{Reacondicionamiento de brackets}

Luego del test de cizallamiento, los brackets guardaron su orden respectivo para cada diente. La resina remanente en la base de los brackets del grupo 1 fue limpiado con microarenado de partículas de óxido de aluminio de $50 \mu \mathrm{m}$. La distancia entre la base del brackets y la punta fue de $8 \mathrm{~mm}$. El microarenador utilizado fue X-blast BX Series 90 de procedencia americana 2004.

La resina remanente en la base de los brackets del grupo 2 fue flameado en un mechero de alcohol y limpiados con un explorador de punta fina.

La resina remanente fue limpiada del esmalte dental con fresa de acero de tungsteno 536L (E.B. Pareja Lecaros S.A. Perú) de 30 hojas no cortante. $\mathrm{Se}$ reacondicionaron los dientes con ácidos fosfórico al $37 \%$ para su segunda adhesión.

Del mismo modo, se repitieron los procedimientos antes mencionadas para una tercera y cuarta adhesión.

\section{RESULTADOS}

En el primer ensayo de cizallamiento, brackets nuevos fueron adheridos a todos los grupos y no se encontró diferencias significativas entre los grupos (Tablas N. ${ }^{\circ} 1$ y 2 )

En el segundo ensayo de cizallamiento, todos los grupos tuvieron una fuerza de adhesión menor que la inicial (Tabla No 3). La mayor fuerza de adhesión lo obtuvo el grupo I $(10.26 \pm 0.79 \mathrm{Mpa})$, seguido por el grupo III $(8.47 \pm 0.49$ $\mathrm{Mpa})$ y el grupo II $(7.52 \pm 1.38 \mathrm{Mpa})$ siendo significativamente entre sí $(\mathrm{P}<$ 0.05) (Tabla N.o 2).

En el tercer ensayo de cizallamiento, los grupos I y II tuvieron una caída significativa en relación a la fuerza de adhesión anterior $(\mathrm{P}<0.05)$. El grupo III no tuvo una caída significativa, sin embargo, la mayor fuerza de adhesión la obtuvo el grupo I. (Tabla N.o 2 y 3 )

Tabla N.o 1. Fuerza de adhesión de los grupos experimentales en $\mathrm{MPa}^{*}$

\begin{tabular}{cccccccccccccc}
\hline & \multicolumn{2}{c}{ Primer ensayo } & \multicolumn{2}{c}{ Segundo ensayo } & & Tercer ensayo & Cuarto ensayo \\
& N & Media \pm DS & Rango & Media \pm DS & Rango & Media \pm DS & Rango & Media \pm DS & Rango & & & & \\
\hline Grupo I & 20 & $11.21 \pm 0.77$ & $9.94-12.73$ & $10.26 \pm 0.79$ & $8.74-11.53$ & $9.36 \pm 1.01$ & $7.56-11.13$ & $6.92 \pm 1.09$ & $5.17-9.54$ \\
Grupo II & 20 & $11.09 \pm 0.55$ & $10.34-11.93$ & $7.52 \pm 1.38$ & $5.57-10.34$ & $6.32 \pm 1.32$ & $4.37-9.54$ & $5.19 \pm 1.13$ & $3.18-6.36$ \\
Grupo III & 20 & $10.86 \pm 0.74$ & $9.54-12.73$ & $8.47 \pm 0.49$ & $7.95-9.54$ & $8.09 \pm 0.79$ & $6.76-9.54$ & $6.82 \pm 1.09$ & $5.17-9.54$ \\
\hline
\end{tabular}

* Fuente: Facultad de Ingeniería Mecánica de la Universidad Nacional de Ingeniería Lima-Perú. 
En el cuarto ensayo de cizallamiento, todos los grupos tuvieron una caída significativa de la fuerza de adhesión en relación a la anterior. La fuerza de ad- hesión del grupo I $(6.92 \pm 1.09 \mathrm{MPa})$ y la del grupo III $(6.82 \pm 1.09 \mathrm{MPa})$ fueron significativamente mayores que la media del grupo II $(5.19 \pm 1.13 \mathrm{MPa})$
$(\mathrm{P}<0.05)$. No hubo diferencias entre el grupo I $(6.92 \pm 1.09 \mathrm{MPa})$ y el grupo III $(6.82 \pm 1.09 \mathrm{MPa})(\mathrm{P}>0.05)$. (Tablas N.o 2 y 3), (Gráfico N.o 1).

Tabla N.o 2. Comparación múltiple de los grupos según los ensayos de cizallamiento.

\begin{tabular}{|c|c|c|c|c|}
\hline GRUPO & Grupo comparac & Dif. de medias & Error std. & Sig.* \\
\hline \multicolumn{5}{|c|}{ Primer ensayo } \\
\hline \multirow{2}{*}{ Arenado } & Nuevo &, 35850 & ,21920 & ,239 \\
\hline & Flameado & ,12000 & ,21920 & ,848 \\
\hline \multirow{2}{*}{ Flameado } & Nuevo & ,23850 & 21920 &, 525 \\
\hline & Arenado &,- 12000 & 21920 & ,848 \\
\hline \multirow{2}{*}{ Nuevo } & Flameado &,- 23850 & ,21920 &, 525 \\
\hline & Arenado &,- 35850 & ,21920 & ,239 \\
\hline \multicolumn{5}{|c|}{ Segundo ensayo } \\
\hline \multirow{2}{*}{ Arenado } & Nuevo & $1,78800^{*}$ & ,30311 & ,000 \\
\hline & Flameado & $2,74050^{*}$ & ,30311 &, 000 \\
\hline \multirow{2}{*}{ Flameado } & Nuevo &,$- 95250^{*}$ & ,30311 & ,007 \\
\hline & Arenado & $-2,74050^{*}$ & ,30311 &, 000 \\
\hline \multirow{2}{*}{ Nuevo } & Flameado & ,95250* & ,30311 &, 007 \\
\hline & Arenado & $-1,78800^{*}$ &, 30311 &, 000 \\
\hline \multicolumn{5}{|c|}{ Tercer ensayo } \\
\hline \multirow{2}{*}{ Arenado } & Nuevo & $1,27100^{*}$ &, 33521 & ,001 \\
\hline & Flameado & $3,03950^{*}$ &, 33521 &, 000 \\
\hline \multirow{2}{*}{ Flameado } & Nuevo & $-1,76850^{*}$ &, 33521 &, 000 \\
\hline & Arenado & $-3,03950^{*}$ &, 33521 &, 000 \\
\hline \multirow{2}{*}{ Nuevo } & Flameado & $1,76850^{*}$ &, 33521 &, 000 \\
\hline & Arenado & $-1,27100^{*}$ &, 33521 & ,001 \\
\hline \multicolumn{5}{|c|}{ Cuarto ensayo } \\
\hline \multirow{2}{*}{ Arenado } & Nuevo &, 10350 & ,34889 & 953 \\
\hline & Flameado & $1,73000^{*}$ & ,34889 &, 000 \\
\hline \multirow{2}{*}{ Flameado } & Nuevo & $-1,62650^{*}$ & ,34889 &, 000 \\
\hline & Arenado & $-1,73000^{*}$ & ,34889 &, 000 \\
\hline \multirow{2}{*}{ Nuevo } & Flameado & $1,62650^{*}$ & ,34889 &, 000 \\
\hline & Arenado &,- 10350 & ,34889 & ,953 \\
\hline
\end{tabular}

$* \mathrm{P}=0,000<0,05$ existe diferencias signifcativas.

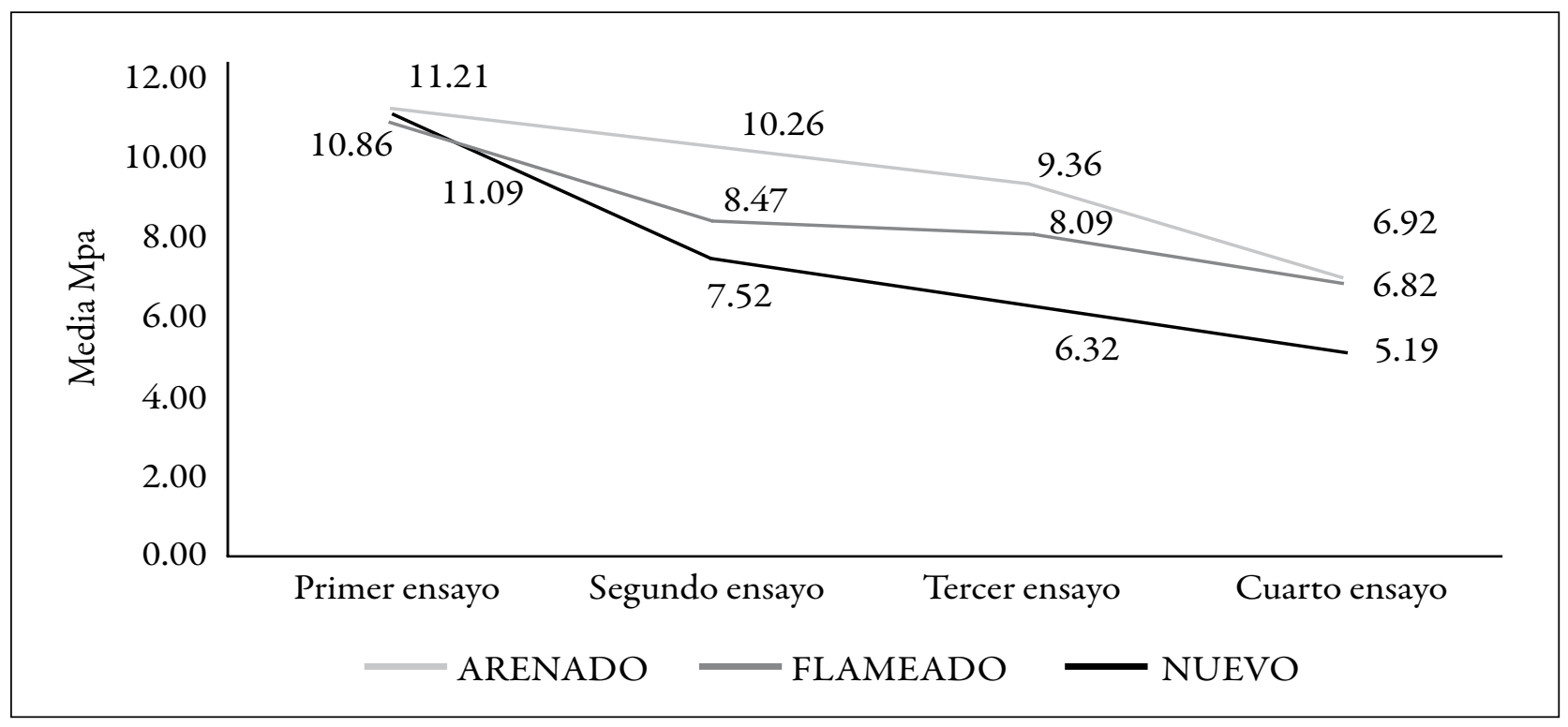

Grafico N.o 1. Fuerza de adhesión de cada grupo según número de ensayos de cizallamiento. 
Tabla N.o 3. Comparación múltiple de los ensayos de cizallamiento de cada grupo.

\begin{tabular}{|c|c|c|c|c|}
\hline Ensayo & Ensayo & Dif. de medias & Error std. & Sig.* \\
\hline \multicolumn{5}{|c|}{ Grupo Arenado } \\
\hline \multirow{3}{*}{$1^{\circ}$ ensayo } & $2^{\circ}$ ensayo & ,95600* & ,29271 & ,009 \\
\hline & $3^{\circ}$ ensayo & $1,85100^{*}$ & ,29271 &, 000 \\
\hline & $4^{\circ}$ ensayo & $4,29400^{*}$ & ,29271 &, 000 \\
\hline \multirow{3}{*}{$2^{\circ}$ ensayo } & $1^{\circ}$ ensayo &,$- 95600^{*}$ & ,29271 & ,009 \\
\hline & $3^{\circ}$ ensayo &, $89500^{*}$ & ,29271 &, 016 \\
\hline & $4^{\circ}$ ensayo & $3,33800^{*}$ & ,29271 &, 000 \\
\hline \multirow{3}{*}{$3^{\circ}$ ensayo } & $1^{\circ}$ ensayo & $-1,85100^{*}$ & ,29271 &, 000 \\
\hline & $2^{\circ}$ ensayo &,$- 89500^{*}$ & ,29271 & 016 \\
\hline & $4^{\circ}$ ensayo & $2,44300^{*}$ & ,29271 &, 000 \\
\hline \multirow{3}{*}{$4^{\circ}$ ensayo } & $1^{\circ}$ ensayo & $-4,29400^{*}$ & ,29271 &, 000 \\
\hline & $2^{\circ}$ ensayo & $-3,33800^{*}$ & ,29271 &, 000 \\
\hline & $3^{\circ}$ ensayo & $-2,44300^{*}$ & ,29271 &, 000 \\
\hline \multicolumn{5}{|c|}{ Grupo Flameado } \\
\hline \multirow{3}{*}{$1^{\circ}$ ensayo } & $2^{\circ}$ ensayo & $3,57650^{*}$ & ,36084 & 000 \\
\hline & $3^{\circ}$ ensayo & $4,77050^{*}$ & ,36084 &, 000 \\
\hline & $4^{\circ}$ ensayo & $5,90400^{*}$ & ,36084 &, 000 \\
\hline \multirow{3}{*}{$2^{\circ}$ ensayo } & $1^{\circ}$ ensayo & $-3,57650^{*}$ & ,36084 &, 000 \\
\hline & $3^{\circ}$ ensayo & $1,19400^{*}$ & ,36084 & ,008 \\
\hline & $4^{\circ}$ ensayo & $2,32750^{*}$ & ,36084 &, 000 \\
\hline \multirow{3}{*}{$3^{\circ}$ ensayo } & $1^{\circ}$ ensayo & $-4,77050^{*}$ & ,36084 & 000 \\
\hline & $2^{\circ}$ ensayo & $-1,19400^{*}$ & ,36084 & ,008 \\
\hline & $4^{\circ}$ ensayo & $1,13350^{*}$ & ,36084 & 013 \\
\hline \multirow{3}{*}{$4^{\circ}$ ensayo } & $1^{\circ}$ ensayo & $-5,90400^{*}$ & ,36084 &, 000 \\
\hline & $2^{\circ}$ ensayo & $-2,32750^{*}$ & ,36084 &, 000 \\
\hline & $3^{\circ}$ ensayo & $-1,13350^{*}$ & ,36084 &, 013 \\
\hline \multicolumn{5}{|c|}{ Grupo Nuevo } \\
\hline \multirow{3}{*}{$1^{\circ}$ ensayo } & $2^{\circ}$ ensayo & $2,38550^{*}$ &, 25422 & 000 \\
\hline & $3^{\circ}$ ensayo & $2,76350^{*}$ &, 25422 &, 000 \\
\hline & $4^{\circ}$ ensayo & $4,03900^{*}$ & ,25422 &, 000 \\
\hline \multirow{3}{*}{$2^{\circ}$ ensayo } & $1^{\circ}$ ensayo & $-2,38550^{*}$ & ,25422 &, 000 \\
\hline & $3^{\circ}$ ensayo & ,37800 &, 25422 &, 450 \\
\hline & $4^{\circ}$ ensayo & $1,65350^{*}$ &, 25422 &, 000 \\
\hline \multirow{3}{*}{$3^{\circ}$ ensayo } & $1^{\circ}$ ensayo & $-2,76350^{*}$ &, 25422 &, 000 \\
\hline & $2^{\circ}$ ensayo &,- 37800 &, 25422 &, 450 \\
\hline & $4^{\circ}$ ensayo & $1,27550^{*}$ &, 25422 &, 000 \\
\hline \multirow{3}{*}{$4^{\circ}$ ensayo } & $1^{\circ}$ ensayo & $-4,03900^{*}$ &, 25422 &, 000 \\
\hline & $2^{\circ}$ ensayo & $-1,65350^{*}$ & ,25422 & 000 \\
\hline & $3^{\circ}$ ensayo & $-1,27550^{*}$ &, 25422 &, 000 \\
\hline
\end{tabular}

$* \mathrm{P}=0,000<0,05$ existe diferencias signifcativas.

\section{DISCUSIÓN}

La mínima fuerza de adhesión clínicamente aceptable no es conocida, sin embargo, Reynolds ${ }^{19}$ y Keizer et al. ${ }^{9}$ sugieren que la adhesión clínica exitosa puede ser lograda con una fuerza de adhesión de 6-10 MPa. Pickett et al. ${ }^{14}$ mencionan que la fuerza de adhesión del bracket debe ser lo suficiente para tolerar las fuerzas biomecánicas y funcionales, pero en un nivel que permita, al final del tratamiento, despegar el bracket sin dañar el esmalte, este último puede ocurrir cuando la fuerza de adhesión supera los $14 \mathrm{MPa}$. Por otro lado, cabe mencionar que la fuerza de adhesión observado in vitro puede ser más alta que la observada in vivo ${ }^{14}$.
La mayoría de estudios miden la fuerza de adhesión en uno o dos ensayos de cizallamiento; sin embargo, en la clínica, puede que se requiera no sólo una readhesión, sino dos o tres.

En el primer ensayo de cizallamiento del presente estudio, nuevos brackets fueron adheridos a todos los grupos para determinar la fuerza de adhesión inicial, no encontrándose diferencias significativas entre los grupos (Tablas N.o 1 y 2). La media de la fuerza de adhesión inicial encontrado fue de $11.05 \pm 0.70$ MPa similar a lo hallado por Fuentes ${ }^{5}$ $10.17 \pm 0.32 \mathrm{MPa}$, quien evaluó el sistema adhesivo No Mix de la American Orthodontic, utilizó el mismo equipo de cizallamiento, pero diferente tipo de brackets que del presente estudio.
En el segundo ensayo de cizallamiento, la fuerza de adhesión de cada grupo disminuyó significativamente en relación a la inicial (Tabla N.o 3). Esto debido posiblemente a que el segundo patrón de grabado ácido del esmalte presentó menor microrretención que el inicial ${ }^{1}$. Sin embargo, los brackets reacondicionados con microarenado (grupo I) presentaron significativamente mayor fuerza de adhesión que los brackets flameados (grupo II) y los brackets nuevos (grupo III) (Tabla N.o 2). Esto puede deberse a que el microarenado no sólo limpia la resina de la base del brackets, sino también aumenta la rugosidad de la base del bracket y, en consecuencia, de su superficie ${ }^{10}$. Estos resultados son similares a trabajos previos que demues- 
tran que el microarenado es un buen método para reacondicionar el bracket, pudiendo tener un comportamiento igual o mejor que los brackets nuevos. Newman et al. ${ }^{13}$ y Ramírez et al. ${ }^{17}$ encontraron que la fuerza de adhesión de brackets arenados fueron mayores a los brackets nuevos. Sonis ${ }^{16}$, Grabouski et al. ${ }^{7}$ Tavares et al. ${ }^{20}$ no encontraron diferencias significativas.

En relación a los brackets nuevos, los resultados del estudio son similares a los encontrados por Bishara ${ }^{2}$, quien evaluó la fuerza de dos adhesivos ortodónticos, adhirió nuevos brackets en cada ensayo de cizallamiento y concluyó que no hubo diferencias significativas en el primer ensayo de cizallamiento, sin embargo, en el segundo ensayo, la fuerza de ambos adhesivos disminuyeron significativamente en relación a la fuerza de adhesión inicial.

Por otro lado, la fuerza de adhesión de los brackets reacondicionados con flameado (grupo II) fue significativamente menor $(\mathrm{p}<0.05)$ que la fuerza inicial y significativamente menor $(\mathrm{p}<0.05)$ a los otros dos grupos (Tabla N.o 2 y 3). Estos resultados coinciden con los encontrados por Regan et al. ${ }^{18}$ y Somporn et al. ${ }^{15}$

En el tercer ensayo de cizallamiento, la fuerza de adhesión del grupo I disminuye significativamente en relación a la fuerza de adhesión del segundo ensayo de cizallamiento; por otro lado, la media del grupo III no tuvo una disminución significativa (Tabla N. ${ }^{o} 3$ ). Esto podría ser resultado del aumento de la coadhesión entre el adhesivo del bracket con los tags de resina remanentes en el esmalte, adicionalmente asociada a una disminución de la microrretención adamantina. Estos resultados no son semejantes con los de Montasser et al. ${ }^{11}$, quienes evaluaron la readhesión de brackets nuevos en tres ensayos de cizallamientos utilizando un sistema de autograbado. Sus resultados señalaron que la fuerza de adhesión inicial fue significativamente menor que la fuerza de adhesión del segundo y tercer ensayo de cizallamiento. Concluyeron que utilizando un sistema de autograbado, la fuerza de adhesión se incrementa conforme al número de ensayos de cizallamientos realizados.

Por otra parte, la fuerza de adhesión del grupo II flameado siguió disminuyendo significativamente (Tabla N.o 3). Esto puede deberse a que la técnica de flameado no elimina completamente la resina de la base del bracket y deja residuos de combustión en la misma ${ }^{18,15}$.
En el cuarto ensayo de cizallamiento, la fuerza de adhesión de todos los grupos tuvo una disminución significativa en relación al tercer ensayo de cizallamiento (Tabla N.o 3). Los grupos I y III presentaron fuerzas de adhesión muy similares sin diferencias significativas ( $\mathrm{p}>$ $0.05)$ y significativamente mayores a los del grupo II $(\mathrm{P}<0.05)$ (Tabla N.o 2). Aparentemente en este cuarto ensayo se produce un estrés máximo de la estructura del esmalte y, en consecuencia, la retención de la base de los brackets deja de ser relevante. Todo hace indicar que si bien existen muchos factores que intervienen en la fuerza de adhesión del bracket como el acondicionamiento del esmalte dental, el sistema adhesivo y el diseño del bracket, estos dos últimos parecen ser recuperables y clínicamente manejables, mientras que el primero podría ser un factor crítico, ya que los cambios ocasionados en la superficie dentaria son irreversibles. En el gráficos 1 se puede observar el comportamiento de la fuerza de adhesión de cada grupo estudiado en relación al número de ensayos de cizallamientos. No se encontró en la literatura estudios con cuatro ensayos de cizallamientos, por los resultados del presente estudio no pueden ser comparados.

NA et al. ${ }^{12}$, Eliades y Brantley ${ }^{4}$ realizaron una revisión de la literatura e hicieron una crítica de la prueba de fuerza de adhesión en ortodoncia. Señalaron que poca atención ha sido puesta en los detalles de los procedimientos y de las pruebas utilizadas; reveló una larga variación en el método utilizado para la prueba de fuerza de adhesión que hacía difícil la comparación de documentos y algunas veces imposible. Sugirió establecer técnicas estándar para una mejor orientación fiable para la clínica ortodóntica.

Al final del estudio se llega a las siguientes conclusiones:

1. A mayor número de veces que se adhiere un bracket sobre la misma superficie del esmalte, menor es la fuerza de adhesión sin importar el tipo de reacondicionamiento o si se adhiere un bracket nuevo.

2. La fuerza de adhesión de brackets reacondicionados con técnica de microarenado alcanzó los valores más altos en relación a los otros grupos; sin embargo, presentó una disminución significativa en relación al número de adhesiones realizadas sobre la misma superficie de esmalte. Esta técnica puede ser recomendada para uso clínico.
3. La fuerza de adhesión de brackets reacondicionados con técnica de flameado presentó los valores más bajos en relación a los otros grupos. Asimismo la fuerza de adhesión disminuyó significativamente en relación al número de adhesiones realizadas sobre la misma superficie del esmalte. Esta técnica no debe ser recomendada para el uso clínico.

4. La fuerza de adhesión de brackets nuevos disminuye significativamente en relación al número de adhesiones realizadas sobre la misma superficie del esmalte.

5. El esmalte dental parece ser un factor determinante en la fuerza de adhesión del bracket, ya que los cambios producidos por cada grabado ácido son irreversibles.

\section{REFERENCIAS BIBLIOGRÁFICAS}

1. Bishara SE, VonWald L, Laffoon JF, Warren JJ. The effect of repeated bonding on the shear bond strength of a composite resin orthodontic adhesive. Angle Orthodontic. 2000; 70:435-441.

2. Bishara SE, John F, Laffoon B, Leigh VonWald BA, John J. The effect of repeated bonding on the shear bond strength of different orthodontic adhesives. American Journal of Orthodontics and Dentofacial Orthopedics 2002; 121:521-5.

3. Buonocore, MG. A simple method of increasing the adhesion of acrylic filling materials to enamel surfaces. Journal of Dental Research. 1955; 34:849-853.

4. Fuentes AA. Estudio comparativo de la adhesión de un ionómero y dos resinas utilizadas para adherir brackets (Tesis para optar el título de Cirujano dentista) Lima: UNMSM; 1999.

5. Eliades T, Brantley WA. The inappropriateness of conventional orthodontic bond strength assessment protocols. Europe Journal of Orthodontic. 2000; 22:13-23

6. Graber TM. Orthodontics. $\mathrm{Cu}-$ rrent Principles and Techniques, 3a edición. USA: Editorial Mosby; 2000:539-624.

7. Grabouski JK, Staley RN, Jakobsen JR. The effect of microetching on the bond strength of metal brackets when bonded to previously bonded teeth: an in vitro study. American 
Journal of Orthodontics and Dentofacial Orthopedics. 1998; 114: 452-460.

8. International Standardization for Organization - Guidance on testing of adhesion to tooth structure. ISO/ TC106/SC 1 N236, Resolution 6 TR 11405; 1994.

9. Keizer S, Ten Cate JM, Arends J. Direct bonding of orthodontic brackets. American Journal Orthodontic and Dentofacial Orthopedic. 1976; 69:318-327.

10. Mattick CR, Hobson RSA. Comparative micro-topographic study of the buccal enamel of different tooth types, Journal of Orthodontics, 2000, 27, 143-148.

11. Montasser MA. Rebonding of Orthodontic Brackets The Angle Orthodontist. 2007 june; 78(3): 531536.

12. Na F, McCabe JF, Buckley JG. A critique of bond strength testing in orthodontics British Journal of Orthodontics. 1994; 21: 33-43.
13. Newman GV, Sun BI, Ha JL, Ozsoylu SA. Adhesion promoters, their effects on the bond strength of metal brackets. American Journal of Orthodontics and Dentofacial Orthopedics. 1995; 108: 237-241.

14. Pickett KL, Sadowsky PL, Jacobson A, Lacefield W. Orthodontic in vivo bond strength: comparison with in vitro results. Angle Orthodontic. 2001; 71: 141-148.

15. Somporn R, et al. The Effect of Reconditioning Methods on Shear Bond Strength of Reused Orthodontic Metal Brackets, Mahidol Dental Journal. 2005; 25(1-3).

16. Sonis AL. Air abrasion of failed bonded metal brackets: a study of shear bond strength and surface characteristics as determined by scanning electron microscopy American Journal of Orthodontics and Dentofacial Orthopedics. $1996 \mathrm{Jul}$; 110(1): 96-8.

17. Ramírez SU, Rubio DM, Valencia FA, García E. Comparación del Esfuerzo a la Tracción de Brackets
Arenados Mediante Óxido de Aluminio Nuevo y Reciclado con y sin Ultrasonido (estudio in vitro) Acta Odontológica Venezolana. 2008; 46 (1).

18. Regan D, LeMasney B, van Noort $\mathrm{R}$. The tensile bond strength of new and rebonded stainless steel orthodontic brackets. Europe Journal of Orthodontic. 1993; 15: 125-135.

19. Reynolds IR. A review of direct orthodontic bonding. British Journal of Orthodontics 1975; 2:171-178.

20. Tavares SW, Consani S, Nouer DF. Shear bond strength of new and recycled brackets to enamel. Brazilian Dental Journal 2006; 17(1): 44-48.

Recibido: 14-11-08

Aceptado para publicación: 12-12-08 